\title{
Cervical Rib Causing Thrombosis of Subclavian Artery
}

\author{
Sharma P, Rasheed I, Ansari MA, ${ }^{3}$ Gurung GS, ${ }^{3}$ Chataut SP ${ }^{4}$ \\ 'Department of Radiology and Imaging, Nepalgunj Medical College Teaching Hospital, Banke, Nepal, 'Department of \\ Radiology, Indira Gandhi Memorial Hospital, Male, Maldives, ${ }^{3}$ Department of Radiology and Imaging, Tribhuvan \\ University Teaching Hospital, Kathmandu, ${ }^{4}$ Department of Radiology, Norvic-Escorts International Hospital, Kathmandu
}

\section{ABSTRACT}

We describe here an unusual case of thrombosis of left subclavian artery in a patient with cervical rib. The patient presented with features of ischaemia of left upper limb. X-ray chest revealed bilateral cervical ribs, longer on the left side. Color Doppler studies showed echogenic thrombus within the left subclavian artery. Angiography revealed complete occlusion of left subclavian artery. Embolectomy was done. She was planned for excision of rib.

Key Words: cervical rib, thrombosis of subclavian artery

\section{INTRODUCTION}

Cervical ribs are anomalies that arise from the lowest cervical vertebrae. It is present in approximately 1-2\% of the population and in $4.5 \%$ of patients with Thoracic outlet syndrome. The expression "thoracic outlet syndrome" (TOS) was first used by Peet to describe patients with neurovascular symptoms relative to possible sites of compression of the neurovascular bundle. ${ }^{1}$ The main symptoms are pain and paraesthesia, which occur spontaneously or are caused or exacerbated by movements that reduce the dimensions of potential compressions spaces.

Arterial complications are rare. Here we report a case of cervical rib associated with thrombosis of left subclavian artery.

\section{CASE REPORT}

A 28 years old female presented with pain and decreased sensation in left upper limb for two months and cold left hand for one month. These symptoms were aggravated for the last 20 days. Physical examination revealed non palpable radial pulse. Other peripheral pulses were normal. Signs of ischemia were present in left upper limb with bluish discoloration of finger tips (Figure 1). All other physical and systemic examinations were unremarkable. Laboratory investigations were also within normal limit. $X$-ray chest revealed bilateral cervical ribs. It was longer on the left side. Doppler study of left upper arm showed a large echogenic thrombus within the left subclavian artery. Small amount of flow was noted at the periphery of the vessel (Figure 2). Left upper limb angiography showed occlusion of left subclavian artery in its distal

Correspondence:

Dr. Prakash Sharma

Department of Radiology and Imaging, Nepalgunj Medical

College Teaching Hospital, Banke, Nepal.

Email: prakashshrm@yahoo.com

Phone:9841264862. 
part with few collaterals (Figure 3). Embolectomy was done and large amount of thrombus was removed from left subclavian artery. Postoperatively, patient regained warmth up to the left wrist joint but developed gangrene of left little finger. Amputation of the gangrenous finger with excision of the cervical rib was planned.

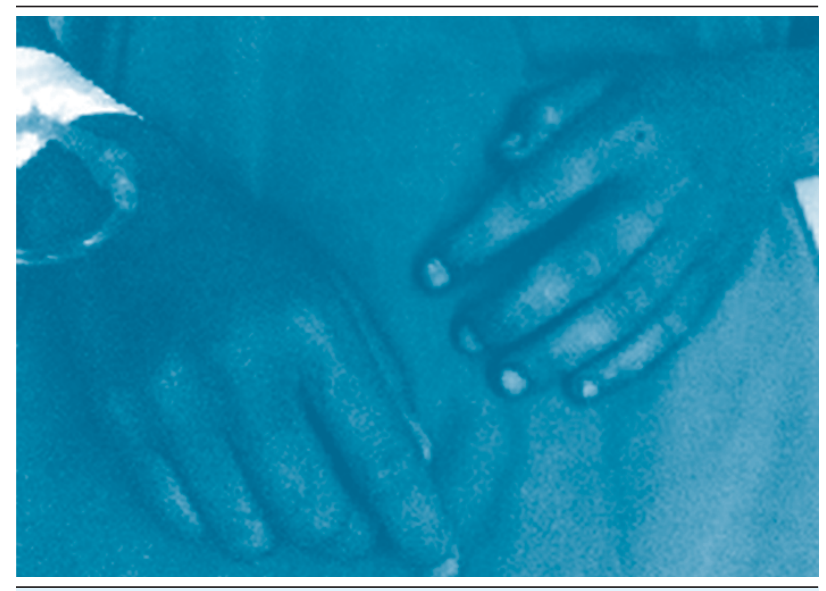

Figure 3. Signs of ischaemia with bluish discolouration of left hand fingers tip

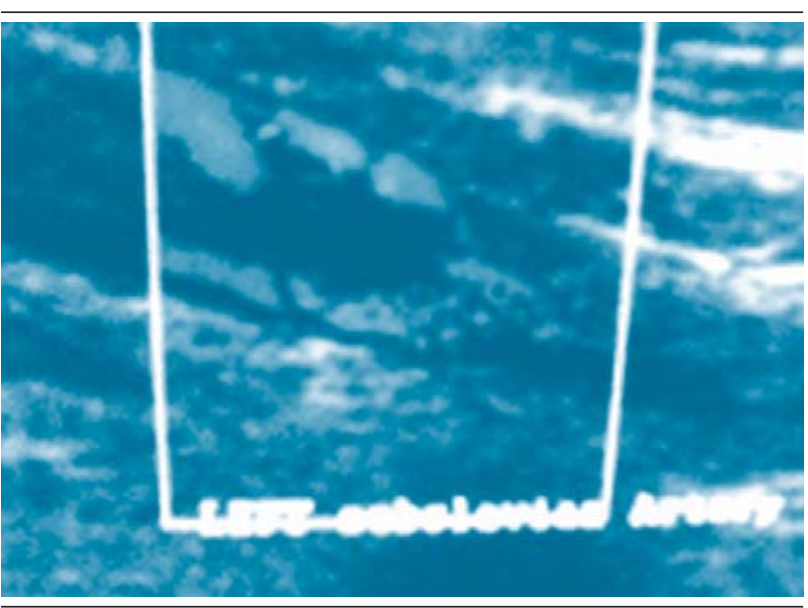

Figure 3. Signs of ischaemia with bluish discolouration of left hand fingers tip

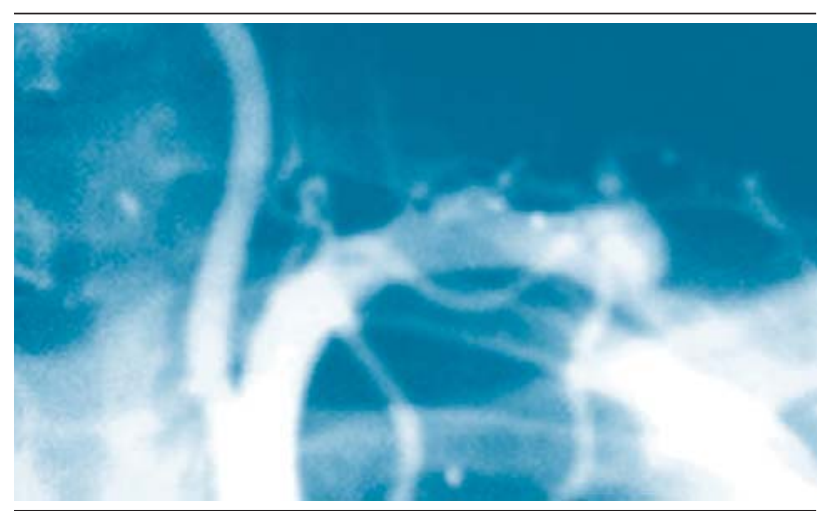

Figure 3. Signs of ischaemia with bluish discolouration of left hand fingers tip

\section{DISCUSSION}

A cervical rib is a rib which arises from the seventh. It is a abnormality located above the normal first rib. Cervical ribs occur in 1-2\% of the population and are commonly bilateral, though often asymmetrical. ${ }^{2}$

Normally, ribs arise from precursor sclerotome cells in the thoracic region. These cells, which are also precursors of a portion of the skull as well as the vertebral bodies, migrate laterally with subsequent development of endochondral ossification centers. Under normal circumstances, the costal processes in the cervical region fail to grow beyond the tip of the vertebral transverse processes. Continued migration of sclerotome precursor cells in the cervical spine region may lead to the formation of a cervical rib. ${ }^{3}$

Cervical rib may be associated with thoracic outlet syndrome. The syndrome involves compression, injury, or irritation to the neurovascular structures at the root of the neck or upper thorax. Compression may involve nerves, including the brachial plexus, usually the lower trunk or medial cord. It could involve compression of the subclavian artery, vein, or both. Thrombosis, embolism, or aneurysm of these vessels is less likely. In our case thrombosis of the subclavian artery was associated with cervical rib.

The occlusion arises secondary to damage to the intima of the artery. This damage can occur as a result of external compression and repetitive stress to the artery. The affected artery demonstrates detectable intimal damage, which is usually secondary to compressive forces exerted by the muscles of the shoulder girdle that compress the artery and bony abnormalities in this area. Intimal disruption precipitates platelet deposition in the area, with resulting thrombosis. ${ }^{4}$

Few cases of thrombosis of subclavian artery are reported in the literature. James TM et al reported thrombosis of subclavian artery in thoracic outlet syndrome. ${ }^{5}$ Fiorentini $C$ et al reported subclavian artery thrombosis in a 45 years old patient. ${ }^{6}$ Fernando $\mathrm{T}$ et al also reported a case of a 37 year-old woman with bilateral cervical rib that developed embolism in the right upper limb from a poststenotic right subclavian artery aneurysm and dilatation of the left subclavian artery, both due to compression.'

Diagnosis of a cervical rib has traditionally been performed by using plain radiographs. However, radiographs limit analysis to ossified bones. Mangrulkar $\mathrm{VH}$ et al used sonography in the diagnosis of cervical rib in children. ${ }^{7}$

Use of noninvasive magnetic resonance angiography has 
been reported to be particularly helpful in the diagnosis of thoracic outlet compression. James TM et al reported a case where magnetic resonance angiography proved to be inadequate in detecting the subclavian aneurysm containing thrombus, which was later noted on the arteriogram. $^{5}$

Computed tomography (CT) may be useful in patients with symptoms suggestive of thoracic outlet syndrome and no osseous abnormalities on plain radiographs. Computed tomographic angiography or magnetic resonance imaging performed in association with postural maneuvers is helpful in analyzing the dynamically induced compression. B-mode and duplex ultrasonography are good supplementary tools for assessment of vessel compression in association with postural maneuvers.

Arteriography is still viewed as the gold standard for delineating artery abnormalities when evaluating the thoracic outlet syndrome. In conclusion, though subclavian artery thrombosis is rare, it should be suspected in patient with cervical rib having vascular symptoms.

\section{REFERENCES}

1. Fernando T, Wander ES, Jose MSS, Domingos MF, Fernando M. Arterial complications of thoracic outlet syndrome. J Vasc Bras. 2008;7(1):150-4.

2. Verschakelen JA. The chest wall, pleura and diaphragm. In: Grainger RG, Allison D, Adam A, Dixon AK eds. Grainger \& Allison's Diagnostic Radiology: A Textbook of Medical Imaging. 4th Edition. London: Churchill Livingstone; 2001. p. 320.

3. Stern JT. Essentials of Gross Anatomy. Philadelphia, PA: FA Davis Co; 1988. p. 51.

4. Mancini MC. Subclavian Artery Thrombosis. [Online]. 2008 [Cited 2009 May 22]. Available from: http://emedicine.medscape.com/article/424673-overview
5. Monica JT, Kwolek CJ, Jupiter JB. Thoracic outlet syndrome with subclavian artery thrombosis undetectable by magnetic resonance angiography. A case report. J Bone Joint Surg Am. 2007 Jul;89(7):1589-93.

6. Fiorentini C, Mattioli S, Graziosi F, Bonfiglioli R, Armstrong TJ, Violante FS. Occupational relevance of subclavian vein thrombosis in association with thoracic outlet syndrome. Scand J Work Environ Health. 2005 Apr;31(2):160-3.

7. Mangrulkar VH, Cohen HL, Dougherty D. Sonography for diagnosis of cervical ribs in children. J Ultrasound Med. 2008 Jul;27(7):1083-6. 